\title{
El diálogo interdisciplinario como estrategia para la integración de las disciplinas humanísticas en el curso Español II
}

Interdisciplinary dialogue as a strategy for the integration of humanistic disciplines in Español II course

Recibido: 12 de octubre de 2021 | Aprobado: 25 de noviembre de 2021

\section{Resumen}

El aprendizaje integrado ha alcanzado importancia en el esfuerzo que están haciendo las universidades para repensar y rediseñar la educación liberal del siglo XXI. En ese sentido, presentamos este estudio que tiene como objetivo ofrecer el relato de experiencia interdisciplinar en la asignatura de Español II (LET-102). La propuesta que ofrecemos ha sido el resultado de la integración de cuatro disciplinas al proceso de comprensión, análisis e interpretación del ensayo "Soy dominicano pero no soy asi", de Manuel Matos Moquete (2005). Como resultado, los estudiantes han tenido la oportunidad de analizar la discursividad textual desde diferentes posturas, enfoques y puntos de vista que ha favorecido significativamente los cuatro pilares de los Estudios Generales presentes en cada una de las asignaturas que conforman este ciclo: investigación, argumentación, lectura y escritura.

Palabras clave: Interdisciplinariedad, transversalidad, aprendizaje integrado, disciplinas humanísticas y educación liberal

1 Ponencia presentada en el XII Simposio Internacional de Estudios Generales (modalidad virtual), Pontificia Universidad Católica Madre y Maestra (PUCMM), República Dominicana, y Red Internacional de Estudios Generales (RIDEG).

* Postgrado y Maestría en Lingüística Aplicada por el Instituto Tecnológico de Santo Domingo (INTEC). Doctor en Estudios del Español: Lingüística y Literatura por la Pontificia Universidad Católica Madre y Maestra (PUCMM). Profesor instructor de la Escuela de Lenguas. Para contactar al autor: joserodriguezn@pucmm.edu.do

ISSN (en línea): 1814-4152 / Sitio web: http://cuaderno.pucmm.edu.do

CÓMO CITAR: Rodríguez Núñez, J. (2022). El diálogo interdisciplinario como estrategia para la integración de las disciplinas humanísticas en el curso Español II. Cuaderno de Pedagogía Universitaria, 19 (37), 69-74. 


\section{Abstract}

Integrated learning has become important in the effort that universities are making to rethink and redesign the liberal education of the twenty-first century. In this sense, we present this study that aims to offer the story of interdisciplinary experience in the subject of Spanish II. The proposal we offer has been the result of the integration of four disciplines to the process of understanding, analysis and interpretation of the essay "I am Dominican but I am not like this", by Manuel Matos Moquete (2005). As a result, students have had the opportunity to analyze textual discursiveness from different positions, approaches and points of view that has significantly favored the four pillars of General Studies present in each of the subjects that make up this cycle: research, argumentation, reading and writing.

Keywords: Interdisciplinarity, transversality, integrated learning, humanistic disciplines and liberal education.

\section{Introducción}

El aprendizaje integrado es una tendencia actual y presente en distintas reformas curriculares, específicamente en la reforma estadounidense de los estudios universitarios revelada por Blackshields (2016) y otros autores. Esto a pesar de las dificultades que encierra el trabajo contra la inercia institucional. No obstante, una serie de iniciativas de reforma durante los últimos años han dirigido su atención hacia el aprendizaje integrado como meta que hay que planear formalmente para una enseñanza interdisciplinar y transdisciplinar, como bien sostiene Jarvis (2001).

Esta modalidad de aprendizaje integrado ha alcanzado importancia en el esfuerzo que están implementando las universidades para repensar y rediseñar la educación liberal del siglo XXI, pues como ha de saberse y comprenderse, en una educación liberal la suma vale más que las partes que la constituyen. En ese sentido, y como bien lo establecen Boni y Pérez (2006), se espera la convicción de que las instituciones de educación superior hagan del aprendizaje integrado un objetivo explícito y, por vía de consecuencia, hacer lo que puedan para ayudar a todos los alumnos para que aprecien su importancia; adquieran las competencias necesarias y tengan oportunidad de practicarlo y desarrollarlo como un apartado formal de su experiencia académica.

No hay que olvidar que la globalización aumenta el ritmo y la complejidad de la vida personal, cívica y social, otorgando mayor peso a nuestras capacidades para la formación continua, el razonamiento práctico y el pensamiento complejo e integral. Por consiguiente, no es posible que las respuestas a la naturaleza multidimensional de las cuestiones sociales y ambientales actuales se hallen en una única disciplina. Para ello, habremos de desarrollar las competencias necesarias para comprender de manera creativa, crítica y constructiva, además de trabajar y enfrentar retos intelectuales, institucionales e internacionales.

Hoy vemos cómo cada vez más en las academias surgen renovadas disciplinas vinculadas a la cognición a través del intercambio interdisciplinar y la transformación de la materia en diversos campos del saber. Es por esto que destacamos propuestas similares a la nuestra como la titulada Estrategias para la formación interdisciplinar en las áreas de Humanidades y Ciencias Sociales y en Artes, Arquitectura y Diseño (Quintá, 2014). En ella se resalta la importancia de una nueva visión del mundo a partir de enfoques de carácter multicultural e intercultural. Estos enfoques promueven conocimientos, valores, actitudes, normas y creencias como alternativa de respuestas satisfactorias a las adversidades que se nos han de presentar en la cotidianidad. Esto conlleva la apertura de medios interactivos e interdisciplinares. Por esto en la referida estrategia surge el multilingüismo en las academias que abarca desde conceptos y estructuras hasta visiones afines de la realidad.

Una segunda propuesta es la presentada por Carvajal (2010) contenida en el artículo Interdisciplinariedad: Desafío para la educación superior y la investigación. La misma surge como respuesta a favorecer nuevas tendencias 
holísticas, integrales e interdisciplinares, dirigidas a la resolución de problemáticas actuales que habrán de solucionarse desde el trabajo colaborativo y la articulación de variadas posturas. En el artículo se describen los diferentes conceptos de abordaje del conocimiento y se plantea la necesidad de hacer cambios en la educación superior y la investigación para abordar estos problemas cada vez más complejos. Se concluye que el desarrollo humano sostenible puede abordarse desde diferentes disciplinas, pero ninguna por sí sola podrá responder a sus principales problemas, siendo muy poco lo que aportan los equipos multidisciplinarios si los expertos de cada disciplina ofrecen una sola visión técnica de su especialidad sin articular el conocimiento con las demás.

Una tercera y última propuesta vinculada a la interdisciplinariedad dialógica la constituye el proyecto presentado por Aronson (2013), quien propone una secuencia temporal de aprendizaje y aplicación que consiste, fundamentalmente, en el antes de la clase, que constituye un primer contacto con el contenido desde distintas disciplinas, y, posteriormente, el desarrollo de la comprensión de conceptos mediane variados mecanismos de aplicación cognitiva como la síntesis, la interpretación y el análisis. Todo esto mediante un intercambio dialógico, basado en la expresión libre, crítica, reflexiva y analítica. De este modo se garantiza la preparación necesaria del estudiantado, tanto dentro como fuera del aula, lo que a la vez conlleva el fomento de espacios de discusión abierta en los que confluyen planteamientos, posturas y puntos de vista.

Es necesario mirar más allá los cambios que nos ofrece el mundo en general. Por eso, las universidades no deben permanecer ajenas a las transformaciones y novedades globales de índole social. En efecto, los estudios del sistema universitario que no van más allá de la educación misma, o incluso de las normas nacionales, están restringiendo su campo de análisis.

En Español Il la integración global de otras áreas y disciplinas es fundamental, no solo por tratarse de una asignatura de Estudios Generales, sino porque además permite que el estudiantado adquiera una comprensión más rica, más abierta y más profunda que le permitirá comprender e interpretar el discurso desde múltiples enfoques y posturas, y así incidir en el desarrollo de su capacidad crítica, analítica y reflexiva.

En consecuencia, el propósito de este artículo es presentar el relato de la implementación de una estrategia integradora con el fin de promover procesos más elevados de interpretación para el desarrollo de una ciudadanía responsable y participativa. La presente propuesta ha sido el resultado de la integración de cuatro disciplinas vinculadas al proceso de comprensión, análisis e interpretación del ensayo "Soy dominicano pero no soy asi", de Manuel Matos Moquete, registrado en la obra del mismo autor, titulada "En la espiral de los tiempos" (2005).

Dada la diversidad y amplitud conceptual, y la heterogeneidad temática del texto, no fue suficiente el análisis desde una o más disciplinas inherentes a la lingüística del texto, como la pragmática o sociolingüística. En ese sentido, ha sido conveniente un análisis más amplio y exhaustivo que involucra, entre otras disciplinas, la historia, por tratarse de un discurso anecdótico narrado desde la época colonial; la psicología, en vista de actitudes y comportamientos creados por el autor; la antropología, dadas las manifestaciones sociales y culturales predominantes en el discurso, y la filosofía, debido al carácter humanístico y reflexivo inherente a la esencia del ser humano, visto desde el utilitarismo como fin último de las campañas publicitarias presentes en el discurso de Moquete.

A continuación, ofrecemos algunas ideas sobre la necesidad de la construcción identitaria a partir de un planteamiento integral; más adelante, explicamos el proceso de cómo fue implementada la estrategia. Por último, ofrecemos la apreciación cualitativa del ejercicio interdisciplinar en el que participaron los estudiantes y las conclusiones que nos ha provocado la implementación de este tipo de experiencia.

1. La construcción de la identidad desde un planteamiento integral e interdisciplinar

Nos disponemos al abordaje de la conceptualización de identidad desde la integración del aprendizaje, la cual ha sido objeto de diversas consideraciones en las ciencias sociales destacándose, principalmente, las provenientes de disciplinas como la literatura, la 
antropología, la filosofía, la historia y la psicología. Dicha conceptualización, según nos indica Mejía (2001), se sitúa desde la comprensión de procesos de desarrollo hasta la integración e influencia sociocultural. Ambos procedimientos confieren integridad e interdisciplinaridad al concepto de identidad que asumimos como fenómeno humano desde el cual el individuo es capaz de establecer diferencias entre él y los demás con los cuales se relaciona socialmente.

El concepto de identidad corresponde a uno de los ejes curriculares, presente en la asignatura de Español II de la Pontificia Universidad Católica Madre y Maestra (PUCMM) que desde el año 2013 forma parte del ciclo académico de los Estudios Generales por tratarse de un curso dirigido a todo el estudiantado de las distintas carreras. Esta asignatura se ofrece durante el segundo cuatrimestre de cada año académico, como bien consta en los planes de estudio de las diversas carreras. En ese sentido, y como bien lo establece la justificación del programa de esta asignatura, presente en el Núcleo del Plan de Estudios del Ciclo de Estudios Generales (PUCMM, 2016), se trata de un curso obligatorio, relacionado con variadas disciplinas como la filosofía, dado que comparte con esta el abordaje de la argumentación como construcción lingüística que busca no demostrar, sino presentar argumentos a favor de determinado punto de vista. De igual forma, se conecta con la sociología debido a la interconexión que se da en todo conglomerado humano a través de la lengua, lo que facilita el entendimiento, la conformación y desarrollo de las sociedades democráticas. Estas y otras disciplinas son integradas al curso de Español II y se desarrollan a través de cuatro actos fundamentales: investigación, argumentación, lectura y escritura.

En efecto, procedimos a llevar a la clase de Español II cuatro profesionales invitados, especialistas en cada una de las disciplinas mencionadas. Los invitados son docentes de la escuela de Ciencias Sociales y Humanidades de esta PUCMM, campus Santo Domingo. Esto con la finalidad de abordar el texto a partir de cada área bajo dos propósitos fundamentales: Analizar el ensayo Soy dominicano pero no soy así desde diversas disciplinas humanísticas para un mayor grado de comprensión del mismo y facilitar la integración de otras áreas en el curso de Español, de modo que se evidencie la interdisciplinariedad como aspecto fundamental de los Estudios Generales.

\section{Implementación de la estrategia}

El proceso de integración fue realizado durante 8 horas de clase, equivalentes a 4 encuentros consecutivos del curso Español II, cuya población estudiantil de segundo cuatrimestre pertenece a distintas carreras y facultades por tratarse de una asignatura de Estudios Generales. A cada sesión asistió un profesor invitado, quien previamente había leído y analizado el ensayo desde su disciplina correspondiente. Los estudiantes, por su parte, habían investigado sobre el objeto de cada disciplina desde la cual sería analizado el ensayo. En ese sentido, cada estudiante debía formular preguntas a los fines de establecerse un diálogo con el docente visitante.

Como etapa final del proceso, cada estudiante elaboró una síntesis comparativa del contenido del ensayo, visto desde las disciplinas analizadas: historia, filosofía, psicología y antropología. De este modo, se expande la comprensión del ensayo mediante la conjugación de elementos implícitos que van más allá de la textualidad discursiva. Finalmente, cada estudiante redactó un artículo basado en una temática del ensayo, enfocada desde las disciplinas antes mencionadas.

En suma, fueron combinados los métodos de comparación, descripción y análisis. El primero consistió en determinar el vínculo que subyace entre el texto y otras realidades que trascienden el contenido del mismo. El segundo método, la descripción, surge a partir del perfil que desde cada área o disciplina se aborda el discurso $\mathrm{y}$, finalmente, desde el método del análisis se descomponen diversos elementos discursivotextuales enmarcados en cada una de las disciplinas relacionadas con el texto.

\section{Resultados}

Culminadas las sesiones, los estudiantes manifestaron:

- Una actitud crítica, autocrítica y reflexiva ante el contenido del ensayo leído. 
- Mayor grado de sensibilidad y compromiso ante la problemática planteada por el autor.

- Una visión más amplia, más abierta y más flexible ante los sucesos narrados.

- Una postura y conclusión distinta y variada a la reflejada en el texto.

- El compromiso de analizar la discursividad textual, oral o escrita, desde diferentes posturas, enfoques y puntos de vista que han favorecido significativamente los cuatro pilares de los Estudios Generales presentes en cada una de las asignaturas que conforman este ciclo: investigación, argumentación, lectura y escritura.

\section{Conclusión}

En definitiva, se ha podido evidenciar un antes y un después durante la lectura del ensayo. El antes, que se proyectaba en una comprensión limitada y parcializada desde la lingüística, la cognición y la pragmática; y el después, que además de lo antes planteado, integra otras variantes que van más allá de unos procesos unidisciplinares de comprensión y producción. En ese sentido, es preciso resaltar las impresiones de los estudiantes, quienes han expresado el cambio experimentado durante el desarrollo de procesos de comprensión del ensayo como género argumentativo y su carácter social orientado desde distintas disciplinas. En efecto, esta dinámica integral les ha permitido y favorecido una visión más amplia, acompañada de procesos inferenciales y cognitivos complejos, tales como el análisis desde diferentes posturas y puntos de vista; además, este ejercicio ha posibilitado la interpretación abierta, analítica, crítica y subjetiva para la construcción de nuevos conocimientos.

Pero lo más importante ha sido la asunción de una conciencia ciudadana e identataria que busca un sentimiento de pertenencia mediante unos cánones de conducta bien definidos y que promueven un ser participativo, cuya ciudadanía no se agote en un dato biológico, filosófico o antropológico; pues la única pertenencia a la que vale la pena apostar es a la de aquel escenario en el que sus miembros participen libremente en un proyecto común, abierto y moralmente legítimo que, en efecto, se perfila integrador.
Lo expuesto en esta propuesta se debe en gran medida al terreno que ha ganado la integración de los procesos de lectura y escritura en la universidad, vistos de forma transversal y, a la vez, interdisciplinar. En este sentido, coincidimos con Carlino (2013) para asumir modos de leer y comprender los escritos desde variadas culturas, disciplinas y contextos. En efecto, estamos llamados a ser conscientes de que en ocasiones las dificultades que presentan nuestros estudiantes al ingresar a los estudios superiores están vinculadas a estas nuevas culturas escritas a las que han de enfrentarse y que corresponden a los distintos campos de estudio. Ante este hecho, debemos favorecer un cambio que potencie el perfil de estudiantes pensadores, analizadores y productores de textos. Pero para ello hace falta una visión más holística, heterogénea y plural ante la interacción con la información y la actitud ante la misma.

Cuando nos hayamos unido como comunidad académica y dejemos a un lado las fronteras disciplinares que se imponen a la construcción de un ciudadano íntegro y proactivo, entonces sí estaremos ante el cambio inminente del ciudadano que requiere la sociedad de hoy y del futuro.

\section{Referencias bibliográficas}

Aronson, N. (2013). Flipped Learning in Higher Education. Upper Saddle River, NJ: Pearson.

Blackshields, D. (2016). Aprendizaje integrado: Investigaciones internacionales y casos prácticos. Narcea, S.A.

Boni, A. y Pérez, A. (2006). Construir la ciudadanía global desde la universidad. Intermón Oxfam ediciones.

Carlino, P. (2013). Escribir, leer y aprender en la universidad. Fondo de cultura económica de Argentina.

Carvajal, Y. (2013). Interdisciplinariedad: Desafío para la educación superior y la investigación. Revista Luna Azul. No. 31, julio-diciembre 2010

Jarvis, P. (2001). Universidades corporativas: Nuevos modelos de aprendizaje en la sociedad global. Narcea, S.A. 
Matos Moquete, M. (2005). En la espiral de los tiempos. Editora Búho.

Mejía, A. (2001). La identidad social y nacional en dominicana: Un análisis psico-social. Editora Taller.

Núcleo del plan de estudios del ciclo de estudios generales (2016) Facultad de Ciencias Sociales, Humanidades y Artes. Pontificia Universidad Católica Madre y Maestra.
Quintá, M.C. (2014). Estrategias para la formación interdisciplinar en las áreas de Humanidades y Ciencias Sociales y en Artes, Arquitectura y Diseño. Redic Innovacesal. Org 